\title{
Evolution of parthenogenesis in the Otiorhynchus scaber complex
}

\author{
JÜRGEN TOMIUK* \& VOLKER LOESCHCKE† \\ Department of Ecology and Genetics, University of Aarhus, Ny Munkegade, DK-8000 Aarhus C, Denmark
}

\begin{abstract}
A new approach for estimating genetic identity values was applied to a data set on allozyme variation in the weevil, Otiorhynchus scaber (Coleoptera: Curculionidae). This species complex contains sexuals and different races of polyploid asexuals. The results were used to reanalyse the phylogenetic relationship among races and to discuss the monophyletic versus the polyphyletic origin of parthenogenesis. A polyphyletic origin of parthenogenesis was suggested based on the assumption of hybridization between related species, and the extinction of an ancestral species. The genetic diversity among the different races was compared. A decreased number of new mutations with increased ploidy level was found among current populations. This correlation was explained by a lower evolutionary rate in polyploid asexual races than in diploid sexual races.
\end{abstract}

Keywords: evolutionary rate, genetic identity, origin of parthenogenesis, polyphyly, polyploidy.

\section{Introduction}

Parthenogenesis has evolved secondarily in many animal and plant groups (White, 1973). When asexual forms arise, these parthenogenetically reproducing clones compete with sexual individuals. Persistant coexistence of closely related sexual and asexual forms requires the sexual population to compensate for the twofold reproductive advantage of clonally reproducing individuals (Maynard Smith, 1978). When explaining the maintenance of sexuality in the population, it is often assumed that asexuals are more sensitive to changing environmental conditions than sexually reproducing populations. The loss of the ability to reorganize the genotypic structure in asexual populations should result in low genetic heterogeneity and disturbed coevolutionary interactions (Glesener \& Tilman, 1978; Jaenike, 1978), whereas sexual populations can produce genetically diverse offspring which may escape extinction in uncertain environments by high genetic adaptability (for review see Williams, 1975; Stearns, 1987).

Suomalainen et al. (1987) discussed the origin of asexuality from a cytological standpoint. On the basis of cytological results, conclusions can be drawn about the evolution of polyploid asexual populations. First,

*Present address: Abteilung für Klinische Genetik, Universität Tübingen, Wilhelmstr. 27, D-7400 Tübingen, Germany.

$\nmid$ To whom correspondence should be addressed. the origin of polyploid individuals may either be due to polyploidization or to interspecific hybridization. Secondly, the kind of parthenogenesis - apomictic or automictic - has consequences for the genetic structure of populations. In apomictically reproducing clones, the heterozygous state is preserved and only mutations add new genotypic variation to the population. Otherwise the existing genetic structure of apomictic populations can be changed only by selection and/or drift. However, various forms of automixis are discussed and usually the existing genetic population structure is unstable (for review see Suomalainen et al., 1987).

In recent studies on asexual species, electrophoresis and molecular genetic methods have been used to reanalyse the phylogeny of asexual forms and to compare these results to morphological studies. Statistical procedures used previously, however, could not be applied directly to allozyme data of polyploid asexual populations. Tomiuk \& Loeschcke (1991) therefore proposed a measure of genetic identity which is applicable to genetic data of both sexual and polyploid asexual populations or species.

In this paper we apply the new genetic identity measure to data on the weevil Otiorhynchus scaber (Coleoptera: Curculionidae), obtained from Suomalainen \& Saura (1973) and Saura et al. (1976). The phylogenetic relationships among different races in this species complex that consists of sexuals and various 
forms of asexuals is reanalysed, and we discuss the origin of the asexual races. Furthermore, we estimate evolutionary rates of races in relation to their common ancestral species, and discuss differences in evolutionary rates as related to the ploidy level of the races.

\section{Materials and methods}

The statistical procedure for estimating genetic identity values in asexual species was described in detail in a recent paper (Tomiuk \& Loeschcke, 1991). The model assumed one ancestral population that passes all its genetic variability to two new subpopulations, and no intraclonal genotypic variation by random chromosomal segregation (i.e. heterozygous clones are stable). New genetic variation is generated only by mutation, and the mutation rate per locus, $\alpha$, was assumed to be constant within populations. We further assumed back mutation to be absent. Thus the number of alleles at a locus may increase with increasing evolutionary time. Based on these assumptions, we defined four genotype classes per locus with frequencies as functions of the degree of homozygosity in the ancestral population, $F_{(0)}$, the genetic identity between current populations and their common ancestral population, $I$, and the ploidy number, $n$. After $t$ generations of divergence from an ancestral population, we expected homozygous individuals which carry only alleles that also existed in the ancestral population with frequency

$F_{(t)}=F_{(0)} I^{n / 2}$,

and heterozygous individuals which also carried only alleles that existed in the ancestral population with frequency

$H_{(t)}=\left(1-F_{\langle 0\rangle}\right) I^{n / 2}$.

Heterozygotes that had one allele in common with the ancestral population and one mutant allele occurred in the frequency $\mathrm{NH}_{(t)}$, where

$N H_{(t)}=1-I^{n / 2}-(1-\sqrt{ } I)^{n}$,

and the fourth genotype class with frequency $N G_{(t)}$ consisted of individuals that had only mutated alleles, where

$N G_{(t)}=(1-\sqrt{ } I)^{n}$.

The degree of heterozygosity in the ancestral population,

$H_{(0)}=1-F_{(0)}$

and the genetic identity values between populations were estimated by least squares comparing the observed and expected frequencies of genotype classes (see Tomiuk \& Loeschcke, 1991).
Enzyme polymorphism was described in three chromosomal races of the weevil Otiorhynchus scaber by Suomalainen \& Saura (1973) and Saura et al. (1976). In their 1973 study, Suomalainen \& Saura sampled individuals around Plesch in Austria. The individuals of this sample were described as diploid sexual individuals. In succeeding studies of this population they observed that the diploid sexual population had been 'contaminated' by tetraploid asexual individuals (A. Saura, personal communication; Suomalainen et al., 1987). However, the contamination of the diploid sexual sample from Plesch ('sex') did not affect the main results and their interpretation presented here (see discussion). Triploid asexual individuals, tri, were collected at two locations in the Austrian Alps (which were pooled in our study), and tetraploid asexual individuals came from five populations, one from Sweden $($ tet $-S)$, Finland $($ tet $-F)$, and Czechoslovakia $($ tet $-C)$, and two from Poland (tet-Br and tet-Bb). Parthenogenesis in the polyploid races of $O$. scaber is apomictic (Suomalainen et al., 1987).

The genotypic frequencies in $O$. scaber given in the above named investigations by Suomalainen, Saura and co-workers were used to estimate genetic identities among the races in order to construct a phylogenetic tree by the unweighted pair group method (UPGMA), as suggested by Sokal \& Sneath (1963). Confidence limits were calculated according to Nei et al. (1985) and Tomiuk \& Graur (1988).

\section{Results}

The mean genetic identity values among the populations of seven races of $O$. scaber ranged from 0.643 to 0.998 (Table 1). Among the asexual races the triploid asexual race evolved most recently from the 'sexual'

Table 1 Mean genetic identity between 'diploid sexual' ('sex'), triploid (tri $\rangle$ and tetraploid asexual races $\langle$ tet $-F$, tet $-S$, tet $-C$, tet-Br, and tet-Bb) of $O$. scaber

\begin{tabular}{|c|c|c|c|c|c|c|}
\hline & $t r i$ & tet $-F$ & tet $-S$ & tet $-C$ & tet $-B r$ & tet-Bb \\
\hline \multirow[t]{2}{*}{ 'sex' } & 0.863 & 0.801 & 0.823 & 0.682 & 0.643 & 0.673 \\
\hline & \pm 0.043 & \pm 0.047 & \pm 0.047 & \pm 0.062 & \pm 0.075 & \pm 0.073 \\
\hline \multirow[t]{2}{*}{$t r i$} & & 0.799 & 0.788 & 0.711 & 0.695 & 0.690 \\
\hline & & \pm 0.065 & \pm 0.065 & \pm 0.077 & \pm 0.086 & \pm 0.083 \\
\hline \multirow[t]{2}{*}{ tet $-F$} & & & 0.998 & 0.929 & 0.929 & 0.940 \\
\hline & & & \pm 0.001 & \pm 0.048 & \pm 0.048 & \pm 0.043 \\
\hline \multirow[t]{2}{*}{ tet $-S$} & & & & 0.917 & 0.919 & 0.937 \\
\hline & & & & \pm 0.049 & \pm 0.047 & \pm 0.044 \\
\hline \multirow[t]{2}{*}{$t e t-C$} & & & & & 0.952 & 0.957 \\
\hline & & & & & \pm 0.044 & \pm 0.031 \\
\hline \multirow[t]{2}{*}{ tet $-B r$} & & & & & & 0.933 \\
\hline & & & & & & \pm 0.046 \\
\hline
\end{tabular}


race $(I=0.86)$. Genetic identities between tetraploid races and the diploid and triploid races $(0.64<I<0.82)$ were lower than those between the 'diploid sexual' and the triploid asexual races. The tetraploid races were closely related to each other $(I \geq 0.92)$. However, there was an inconsistency between the clusters: the Polish and Czechoslovakian tetraploid populations have diverged earlier from the 'diploid'-triploid lineage $(0.64<I<0.71)$ than the Swedish and Finnish populations $(0.79<I<0.82)$, but the two tetraploid groups were closely related $(0.92<I<0.94$, Fig. 1$)$.

We estimated confidence limits for the branching points in our phylogenetic trees constructed by the UPGMA. The estimation procedure suggested by Nei et al. (1985) was simplified by our approach to estimating genetic identities. For example, we estimated directly the confidence limits of hierarchical branching points when single locus identities between species within different clusters were pooled, and the mean genetic identity among clusters and their confidence limits were obtained by the formulae given by Tomiuk \& Graur (1988). Thus problems of estimating covariances among hierachical branching points which occur with other estimates of genetic identity (e.g. Nei et al., 1985; Pamilo, 1990) were avoided.

The identity values obtained from the different inter-race comparisons were highly consistent when excluding either the group containing the tetraploids from Poland and Czechoslovakia (Fig. 2a) or excluding the tetraploids, tet-S and tet-F (Fig. $2 \mathrm{~b}$ ). The northern European tetraploids, tet- $F$ and tet- $S$, have separated recently from each other (Figs $2 \mathrm{a}$ and $\mathrm{c}$ ). The hypothesis of a monophyletic origin of the parthenogenetic races, tri, tet-S, and tet-F, could not be rejected by the data at a significance level of $P=5$ per cent (Fig. 2a). On the other hand, the cluster containing the 'sexual' and triploid races was separated from the Central European tetraploids $(P<5$ per cent, Fig. $2 \mathrm{~b}$ ).

The method of Tomiuk \& Loeschcke (1991) also allowed an estimation of the genetic identity value between each race and their common ancestral pop-

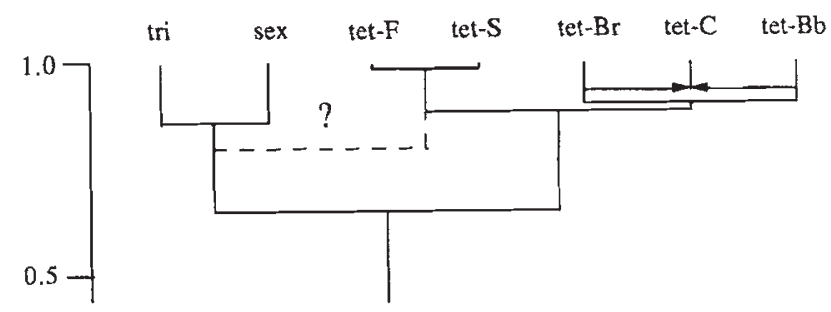

Fig. 1 Phylogenetic tree constructed from genetic identity values between seven populations of $O$. scaber (data from Table 1). For further explanation see text. ulations (Table 2). The genetic identity value, $I$, is closely correlated to the evolutionary rate, $\alpha t$, which is the number of mutations per locus added to the population during evolutionary time (see Tomiuk \& Loeschcke, 1991). When the genetic identity value between one race and the common ancestor is lower than the genetic identity between the other race and its common ancestor, we conclude that the evolutionary rate is higher in the first lineage. Differences in the inferred evolutionary rates between the 'sexual' and triploid races or among the tetraploid races were typically smaller than in the 'sexual' and triploid races (Table 2).

The expected and observed degrees of heterozygosity of the current 'sexual' population and observed values of the current asexual populations were obtained from Suomalainen \& Saura (1973) and Saura et al. (1976). No reliable estimates of the
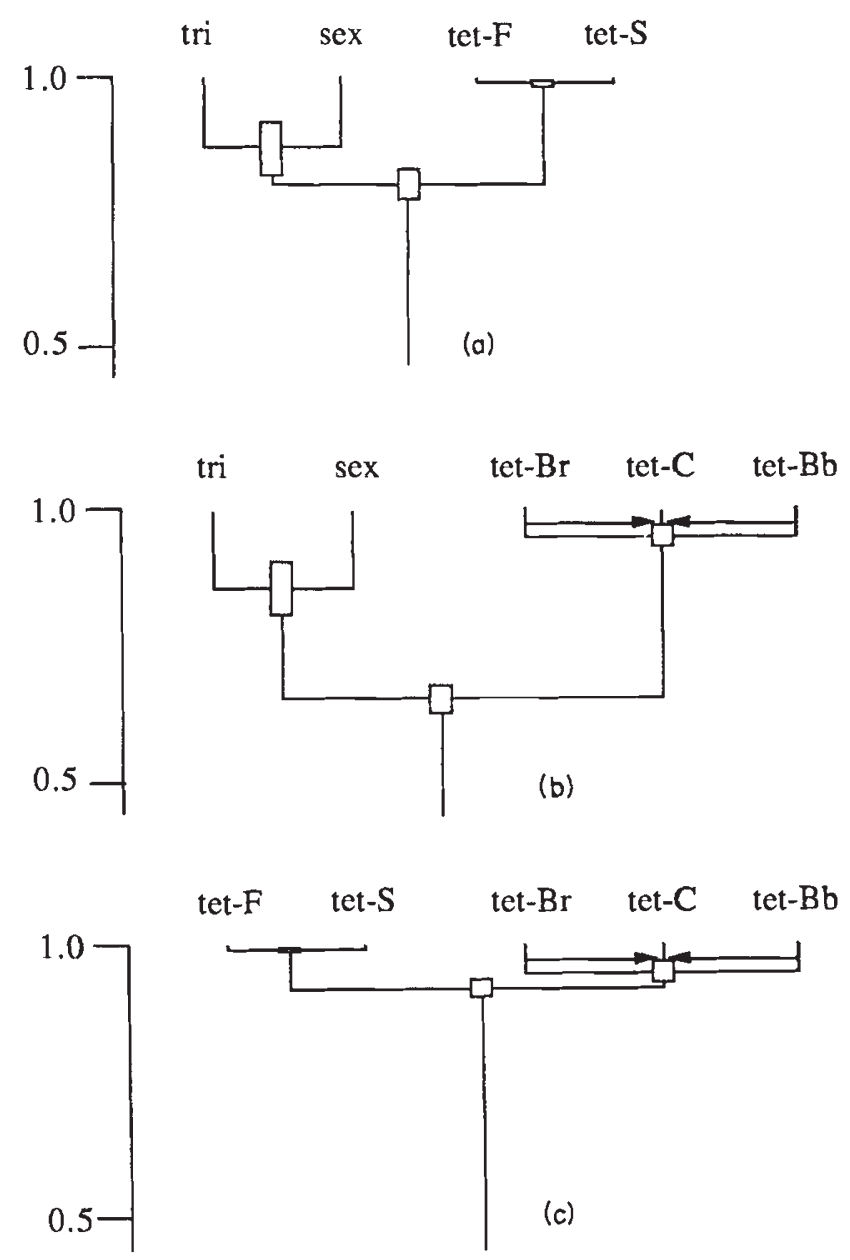

Fig. 2 Phylogenetic trees constructed from genetic identity values: (a) amoung 'sexual', triploid and northen European tetraploid races, (b) among 'sexual', triploid, and central European tetraploid races, (c) among tetraploid races of $O$. scaber (data from Table 1). Sampling errors are indicated by open rectangles. For further explanation see text. 
Table 2 Genetic identity values between ancestral and current populations in the $O$. scaber complex obtained from comparisons of two species

\begin{tabular}{|c|c|c|c|c|}
\hline \multirow[b]{2}{*}{ Species } & \multicolumn{4}{|l|}{ Ploidy level } \\
\hline & 2 & 3 & 4 & 4 \\
\hline 'sex'/tri & $0.871 \pm 0.040$ & $0.907 \pm 0.044$ & & \\
\hline 'sex'/tet-F & $0.785 \pm 0.059$ & & 0.884 & $=0.059$ \\
\hline sex'/tet-S & $0.796 \pm 0.057$ & & 0.910 & $=0.054$ \\
\hline 'sex'/tet-C & $0.641 \pm 0.082$ & & 0.846 & $=0.072$ \\
\hline 'sex'/tet-Br & $0.629 \pm 0.092$ & & 0.773 & $=0.083$ \\
\hline 'sex'/tet-Bb & $0.638 \pm 0.087$ & & 0.815 & $=0.083$ \\
\hline tri/tet-F & & $0.794 \pm 0.077$ & 0.924 & $=0.050$ \\
\hline tri/tet-S & & $0.717 \pm 0.084$ & 0.925 & $=0.051$ \\
\hline tri/tet-C & & $0.648 \pm 0.096$ & 0.901 & $=0.067$ \\
\hline tri/tet-Br & & $0.660 \pm 0.101$ & 0.845 & $=0.079$ \\
\hline tri/tet-Bb & & $0.647 \pm 0.097$ & 0.913 & $=0.060$ \\
\hline tet-F/tet-S & & & $0.997 \pm 0.002$ & $0.999 \pm 0.001$ \\
\hline tet-F/tet-C & & & $0.912 \pm 0.056$ & $0.959 \pm 0.041$ \\
\hline tet-F/tet-Br & & & $0.913 \pm 0.057$ & $0.958 \pm 0.041$ \\
\hline tet-F/tet-Bb & & & $0.928 \pm 0.047$ & $0.957 \pm 0.041$ \\
\hline tet-S/tet-C & & & $0.914 \pm 0.056$ & $0.936 \pm 0.046$ \\
\hline tet-S/tet-Br & & & $0.919 \pm 0.055$ & $0.925 \pm 0.052$ \\
\hline tet-S/tet-Bb & & & $0.920 \pm 0.053$ & $0.954 \pm 0.043$ \\
\hline tet $-C /$ tet $-B r$ & & & $0.957 \pm 0.044$ & $0.947 \pm 0.044$ \\
\hline tet-C/tet-Bb & & & $0.975 \pm 0.025$ & $0.957 \pm 0.044$ \\
\hline tet $-B r / t e t-B b$ & & & $0.915 \pm 0.052$ & $0.957 \pm 0.044$ \\
\hline
\end{tabular}

expected degrees of heterozygosity could be given for the polyploid races. The estimated degrees of heterozygosity of the common ancestral population obtained from comparisons between races were compared with those of the current population (Table 3). The observed and expected heterozygosity values were of the same order of magnitude for the 'sexual' and triploid races. The degrees of heterozygosity for tetraploid races, however, were higher than those of the diploid and triploid races, but sampling errors were large, and the null hypothesis - that the observed and expected degrees of heterozygosity of all races are within the same range independent of the ploidy level or mode of reproduction - could not be rejected. All estimated degrees of heterozygosity obtained from race comparisons that included diploid and triploid populations were considerably smaller than those for the current diploid or triploid populations $(P<5$ per cent). The degrees of heterozygosity of the current tetraploid races and their tetraploid ancestral populations, however, were more similar, and not significantly different, with $0.37 \leq H \leq 0.46$.

\section{Discussion}

The analysis of the phylogeny of asexual species is methodologically difficult compared to the study of sexual species. In sexual species, we hypothesize that species have evolved continuously from one ancestral population, and we can compare the genetic structure of current populations on the basis of some more or less restrictive assumptions. For the analysis of the phylogeny of asexuals, knowledge of the mode of parthenogenetic reproduction is required. The mechanism of maturation division of the egg has consequences for changes in the genotypic structure of asexual populations with time. Additionally, the course of polyploidization is dependent on the cell division mechanism. From cytological observations we know that in some species, higher ploidy levels have been induced by genome duplication (autopolyploidy), whereas in others, higher ploidy levels result from fertilization of normally asexual individuals by closely related males of 'sexual' species (diploid $(2 n)+1 n \rightarrow$ triploid $(3 n)+1 n \rightarrow$ tetraploid (4n) etc.; for discussion see White, 1978). Alternatively, asexual species may be hybrids between related sexual species (e.g. Vrijenhoek, 1984; Parker et $a l ., 1989)$. The various possible origins of asexuality affect the potential genetic variation in the new species. If monophyly (in a strict sense) is assumed, then only one individual is the ancestor of the existent population, but a polyphyletic origin from several ancestral individuals could provide more variation. Beyond that it is difficult to predict how much genetic variability has 
Table 3 Degrees of heterozygosity of different races of $O$. scaber. Observed (obs.) and expected (exp.) values of the current 'sexual' population, and observed values of the current asexual populations are given in the diagonal. The estimated degree of heterozygosity (exp.) in the ancestral population which is obtained from comparisons between races is given below the diagonal

\begin{tabular}{|c|c|c|c|c|c|c|c|}
\hline Species & 'sex' & tri & tet $-F$ & tet $-S$ & tet-C & $t e t-B r$ & $t e t-B b$ \\
\hline \multicolumn{8}{|l|}{ 'sex' } \\
\hline obs. & $\begin{array}{r}0.318 \\
\pm 0.058\end{array}$ & & & & & & \\
\hline exp. & 0.294 & & & & & & \\
\hline & \pm 0.053 & & & & & & \\
\hline \multicolumn{8}{|l|}{ tri } \\
\hline obs. & & $\begin{array}{r}0.321 \\
\pm 0.084\end{array}$ & & & & & \\
\hline exp. & $\begin{array}{r}0.189 \\
\pm 0.049\end{array}$ & & & & & & \\
\hline \multicolumn{8}{|l|}{ tet $-F$} \\
\hline obs. & & & $\begin{array}{r}0.384 \\
\pm 0.111\end{array}$ & & & & \\
\hline exp. & $\begin{array}{r}0.161 \\
\pm 0.062\end{array}$ & $\begin{array}{r}0.252 \\
\pm 0.090\end{array}$ & & & & & \\
\hline \multicolumn{8}{|c|}{$10.002 \quad-0.030$} \\
\hline obs. & & & & $\begin{array}{r}0.370 \\
\pm 0.111\end{array}$ & & & \\
\hline exp. & $\begin{array}{r}0.163 \\
\pm 0.061\end{array}$ & $\begin{array}{r}0.245 \\
\pm 0.088\end{array}$ & $\begin{array}{r}0.355 \\
\pm 0.108\end{array}$ & & & & \\
\hline \multicolumn{8}{|c|}{$-0.000-0.000-0.000$} \\
\hline obs. & & & & & $\begin{array}{r}0.412 \\
\pm 0.123\end{array}$ & & \\
\hline exp. & $\begin{array}{r}0.151 \\
\pm 0.068\end{array}$ & $\begin{array}{r}0.238 \\
\pm 0.097\end{array}$ & $\begin{array}{r}0.361 \\
\pm 0.113\end{array}$ & $\begin{array}{r}0.357 \\
\pm 0.112\end{array}$ & & & \\
\hline $\begin{array}{l}\text { tet-Br } \\
\text { obs. }\end{array}$ & & & & & & $\begin{array}{r}0.456 \\
\pm 0.125\end{array}$ & \\
\hline exp. & $\begin{array}{r}0.126 \\
\pm 0.060\end{array}$ & $\begin{array}{r}0.221 \\
\pm 0.091\end{array}$ & $\begin{array}{r}0.370 \\
\pm 0.112\end{array}$ & $\begin{array}{r}0.366 \\
\pm 0.110\end{array}$ & $\begin{array}{r}0.382 \\
\pm 0.118\end{array}$ & & \\
\hline $\begin{array}{l}\text { tet }-B b \\
\text { obs. }\end{array}$ & & & & & & & $\begin{array}{r}0.406 \\
\pm 0.123\end{array}$ \\
\hline exp. & $\begin{array}{r}0.129 \\
\pm 0.059\end{array}$ & $\begin{array}{r}0.225 \\
\pm 0.094\end{array}$ & $\begin{array}{r}0.348 \\
\pm 0.109\end{array}$ & $\begin{array}{r}0.346 \\
\pm 0.109\end{array}$ & $\begin{array}{r}0.386 \\
\pm 0.118\end{array}$ & $\begin{array}{r}0.295 \\
\pm 0.106\end{array}$ & \\
\hline
\end{tabular}

been contributed to the asexual lineage by hypothesized ancestral species. If the ancestral species that was involved in the evolutionary process became extinct, then the phylogeny based on an estimation of genetic identities between existent forms can be biased.

When we compare the genetic identity values among races within the $O$. scaber species complex, relations among races appear to be similar to the moth group Solenobia triquetrella (Lepidoptera: Psychidae) (Lokki et al, 1975; Tomiuk \& Loeschcke, 1991). Races within clusters show high genetic identity values, but some races belonging to different clusters are also closely related. Such patterns could be explained as samples that contain a mixture of races with different ploidy levels as known for the sample from Plesch ('sex'), but the inconsistency remains even if the triploid and tetraploid races only were considered. By accepting the concept of an increasing ploidy level $(+1 n)$ (Suomalainen et al., 1987), the confusing pattern of inter-race relationships can be explained. If a missing sexual sister-species that has become extinct is assumed, then the origin of triploid individuals of $O$. scaber can be explained by hybridization between the two sexual lineages. Continuing this reasoning we suggest that the Polish and Czechoslovakian tetraploid races were formed by fertilization of ancestral individ- 


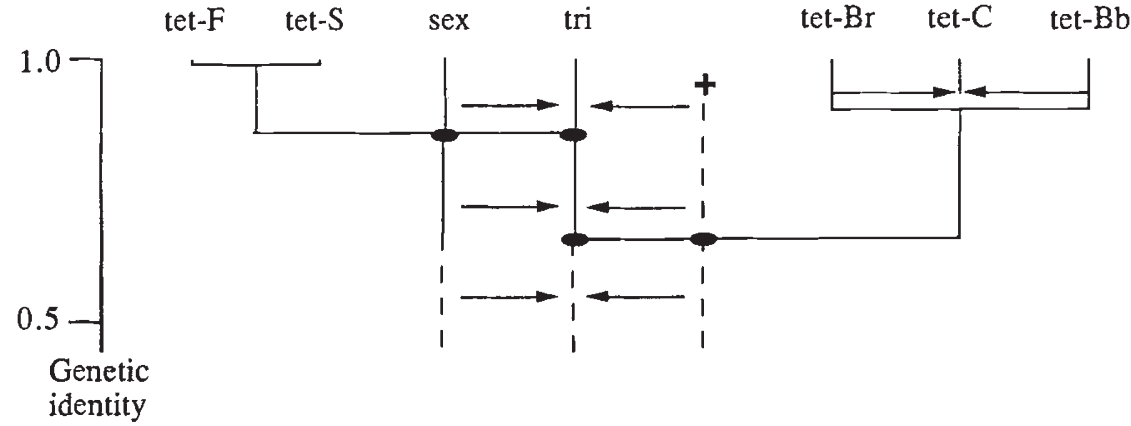

Fig. 3 Phylogenetic tree constructed from genetic identity values between seven populations of $O$. scaber assuming an additional species that has gone extinct $(+)$. uals of the common triploid race by the extinct sexual species, whereas the Scandinavian tetraploids originated later by fertilization of the ancestral individuals of the common triploid race by the current sexual race (Fig. 3). Furthermore, the estimated time since the occurrence of triploid races of $O$. scaber must be smaller than that of tetraploid races. This hypothesis is supported by the data given in Table 3. Evolutionary changes are slower in tetraploid races than in 'sexual' and triploid races and therefore higher genetic identity values among tetraploid races are expected, even with polyphyletic origin. This difference cannot be an artefact caused by the 'contaminated' sample from Plesch, as mixing data from diploid and tetraploid races should reduce differences among races. Thus the application of our method to enzyme data on the species complex $O$. scaber supports the hypothesis of a polyphyletic origin for this group, contrary to the hypothesis of Suomalainen et al. (1987) who suggested a monophyletic origin.

In $S$. triquetrella the estimated degrees of heterozygosity were not significantly different either among different ancestral populations or among species with different degrees of polyploidy (Lokki et al., 1975; Tomiuk \& Loeschcke, 1991). In O. scaber there is also a high consistency among current populations of varying ploidy level for the expected and observed degrees of heterozygosity.

Estimates of evolutionary rates have been obtained in previous studies by comparisons of in-group species to an out-group species. Our approach of estimating genetic identities, however, allows a direct estimate of evolutionary rates from comparisons of two related species or races. Despite a possible bias of the estimates from the sample taken at Plesch, the results from protein data on different races of $O$. scaber suggest that evolutionary rates of polyploid races are lower than those of diploid races. Similarly, Suomalainen (1961) concluded from morphometrical studies on $O$. scaber that evolution has slowed down in parthenogenetic polyploid populations. Lower incorporation of mutations with increasing ploidy level in apomictic species may be explained by more disturbed intragenomic interactions (see e.g. Crow \& Temin, 1964; Templeton et al., 1976). In sexual species, genes function in different genetic backgrounds due to recombination, whereas in apomictic species, a mutated gene can destroy favourable gene combinations, thus reducing the number of new alleles. The high degree of heterozygosity in asexual races is not due to 'new' mutations, but caused by high frequencies of heterozygous individuals carrying 'ancestral' alleles that also are present in the sexual-sister form as observed in S. triquetrella (Lokki et al., 1975) and O. scaber (Suomalainen et al., 1973; Saura et al., 1976).

These restrictions to the incorporation of new mutations reduce the potential for asexuals to adapt to changing environments. However, in stable or predictable environments, the most fit clonal-line may outcompete sexual forms through a higher degree of heterozygosity and more rapid reproduction rate even with low initial population.

\section{Acknowledgments}

We are grateful to Drs J. S. F. Barker, J. J. Boomsma, B. Christensen, F. B. Christiansen, R. A. Krebs, P. Pamilo, E. D. Parker, A. Saura and K. Wöhrmann for discussions of the concept and/or comments on earlier drafts of the manuscript, to Claus Hedegaard for his help in preparing the Figures and to Lars Witting for polishing the computer program which is available on request. The final version of the manuscript was written while V.L. was Research Associate at the Department of Animal Sciences, University of New England, Armidale, N.S.W. He is grateful to Professor Barker for the hospitality received and to the Danish Research Council for supporting his stay.

\section{References}

CROW, J. F. AND TEMIN, R. C. 1964. Evidence for the partial dominance of recessive alleles in natural populations of Drosophila. Am. Nat., 98, 21-33. 
GLESENER, R. R. AND TILMAN, D. 1978. Sexuality and the components of environmental uncertainty: clues from geographic parthenogenesis in terrestrial animals. Am. Nat., 112, 659-673.

JAENIKE, J. 1978. An hypothesis to account for the maintenance of sex within populations. Evol. Theory, 3,191194.

LOKKI, J., SUOMALAINEN, E., SAURA, A. AND LANKINEN, P. 1975. Genetic polymorphism and evolution in parthenogenetic animals. II. Diploid and polyploid Solenobia triquetrella (Lepidoptera: Psychidae). Genetics, 79, 513-525.

MAYNARD SMIth, J. 1978. The Evolution of Sex. Cambridge University Press, Cambridge.

NEI, M., STEPHENS, J. AND SAITOU, N. 1985. Methods for computing the standard errors of branching points in an evolutionary tree and their application to molecular data from humans apes. Molec. Biol. Evol., 2, 66-85.

PAMILo, P. 1990. Statistical test of phenograms based on genetic distances. Evolution, 44, 689-697.

PARKER, JR., E. D., WALKER, J. M. AND PAULISSEN, M. A. 1989. Conal diversity in Cnemidophorus: Ecological and morphological consequences. In: Dawley, R. M. and Bogart, J. B. (eds) Evolution and Ecology of Unisexual Vertebrates, Bulletin 466, New York State Museum, Albany, New York, pp. 72-86.

SAURA, A., LOKKI, J., LANKINEN, P. AND SUOMALAINEN, E. 1976. Genetic polymorphism and evolution in parthenogenetic animals. III. Tetraploid Otiorhynchus scaber (Coleoptera: Curculionidae). Hereditas, 82, 79-100.

SOKAL, R. R. AND SNEATH, P. H. A. 1963. Principles of Numerical Taxonomy. Freeman, San Francisco.
StEArns, s. C. 1987. The Evolution of Sex and its Consequences. Birkhäuser Verlag, Basel, Boston.

SUOMALAINEN, E. 1961. On morphological differences and evolution of different polyploid parthenogenetic weevil populations. Hereditas, 47, 309-341.

SUOMALAINEN, E. AND SAURA, A. 1973. Genetic polymorphism and evolution in parthenogenetic animals. I. Polyploid Curculionidae. Genetics, 74, 489-508.

suomalainen, E., SAURA, A. AND LOKKI, J. 1987. Cytology and Evolution in Parthenogenesis. CRC Press, Boca Raton, Florida.

TEMPLETON, A. R., CARSON, H. L. AND SING, CH.F. 1976. The population genetics of parthenogenetic strains of Drosophila mercatorum. II. The capacity for parthenogenesis in a natural bisexual population. Genetics, 82, 527-542.

TOMIUK, J. AND GRAUR, D. 1988. Nei's modified identity and distance measure and their sampling variances. Sys. Zool., 37, 156-162.

TOMIUK, J. AND LOESCHCKE, v. 1991. A new measure of genetic identity between populations of asexual and sexual species. Evolution, 45, 1685-1694.

VRIJENHOEK, R. C. 1984. Ecological differentiation among clones: the frozen variation model. In: Wöhrmann, K. and Loeschcke, V. (eds) Population Biology and Evolution, Springer Verlag, Heidelberg, New York, pp. 217-231.

white, M. J. D. 1973. Animal Cytology and Evolution, 3rd edn. Cambridge University Press, Cambridge.

white, M. J. D. 1978. Modes of Speciation. Freeman, San Francisco.

WILliams, G. C. 1975. Sex and Evolution. Princeton University Press, Princeton, New Jersey. 